Advances in Fuzzy Mathematics.

ISSN 0973-533X Volume 13, Number 1 (2018), pp. 25-38

(C) Research India Publications

https://dx.doi.org/10.37622/AFM/13.1.2018.25-38

\title{
Some Properties of Fuzzy Soft B-Open Sets and Fuzzy Soft B-Continuous Functions in Fuzzy Soft Topological Spaces
}

\author{
Sandhya G V and *Anil P N \\ Department of Mathematics, Global Academy of Technology, \\ Bengaluru, Karnataka, India. \\ Corresponding Author: *Dr. Anil P N
}

\begin{abstract}
In this paper we introduce fuzzy soft semi pre-open set and studied its relation with fuzzy soft b-open set and also b-continuous functions in fuzzy soft topological spaces and investigate some of their properties. Further generalized b-closed set in fuzzy soft topology, fuzzy soft generalized bcontinuous functions and fuzzy soft generalized b-irresolute functions and fuzzy soft $b^{*}$ - continuous functions have been introduced and studied few of their properties.
\end{abstract}

Keywords: Fuzzy soft Topology, Fuzzy soft semi-pre-open set, Fuzzy soft bcontinuous functions, Fuzzy soft generalized b-continuous functions, Fuzzy soft generalized b-irresolute functions, fuzzy soft $b^{*}$ - continuous functions.

\section{INTRODUCTION}

In recent times, the problems in the field of Engineering, Physics, Social sciences and Medical sciences etc.. involving uncertainities cannot be dealt with crisp data. The general mathematical tool for dealing with uncertainities known as Fuzzy set was introduced by zadeh [15] in 1965. Fuzzy topology was introduced by Chang [4] in 1968. To overcome the existing difficulties in Fuzzy set theory, Molodtsov[10] introduced Soft sets in 1999. The theory of soft sets can be successfully applied in several directions such as Game theory, Riemann integration, Smoothness of functions, Probability theory etc...The hybridisation of Fuzzy set and soft set called Fuzzy soft set was introduced by Maji et..al [9]. The notion of topological structure of Fuzzy soft sets was introduced by Tanay and Kandemir [13] in 2011. And studied further by Varol and Aygun [14] , S Roy and Samanta [11] and many more authors [6],[5]. Mappings of fuzzy soft classes are studied by A.Kharal and B.Ahmad [8]. The concept of fuzzy soft semi open set was introduced by A.Kandil et.al [8] in 2014. 
The concept of fuzzy soft pre-open and regular open sets was introduced by Sabir Hussain in 2016[12]. A new form of fuzzy subset called fuzzy b-open set was introduced by S.S Benchalli and K.Jenifer[2]. S. S.Benchalli and Jenifer J.Karnel also introduced Fuzzy gb-Continuous Maps in Fuzzy Topological Spaces[3]. The concept of fuzzy soft b-open sets was introduced by Anil P.N [1] in 2016.

In this paper a new form of set known as fuzzy soft semi-pre-open set and a new class of continuous functions known as b-continuous functions, generalised b-continuous, generalised $b$-irresolute functions and $b^{*}$ - continuous functions in Fuzzy soft topology are introduced and few of their properties are studied.

\section{PRELIMINARIES}

Definition $2.1^{[9]}$ : Let $\mathrm{X}$ be the initial universe and $\mathrm{E}$ be the set of parameters. $I^{x}$ be the set of all fuzzy sets on X. Let $A \subseteq E$ and $f: A \rightarrow I^{x}$. A pair $(f, A)$ is called fuzzy soft set over X. It is also denoted by $f_{A}$. i.e for every $a \in A, f(a)=f_{a}: X \rightarrow I$ is a fuzzy set on $\mathrm{X}$.

Definition $2.2^{[13]}$ : Let $\tau$ be a collection of all fuzzy soft sets over a universe $\mathrm{X}$ with a fixed set of parameter set $\mathrm{E}$, then a triplet $(X, \tau, E)$ is called fuzzy soft topological space[FSTS] if it satisfies the following axioms.

(i) $\tilde{0}_{E}, \tilde{1}_{E} \in \tau$

(ii)Arbitrary union of members of $\tau$ is a member of $\tau$.

(iii) Finite intersection of members of $\tau$ is a member of $\tau$.

Every member of $\tau$ is called fuzzy soft open set. i.e a fuzzy soft set $f_{A} \in \tau$ is called fuzzy soft open set in $\mathrm{X}$ and its complement $1-f_{A}$ is called fuzzy soft closed set.

Definition $2.3^{[14]}$ : The intersection of all fuzzy soft closed super sets of $f_{A}$ is called fuzzy soft closure of $f_{A}$, denoted by,

$\operatorname{Fscl}\left(f_{A}\right)=\bigcap\left\{h_{D}, h_{D}\right.$ is fuzzy soft closed set and $\left.f_{A} \subseteq h_{D}\right\}$

Definition $2.4^{[14]}$ : The union of all fuzzy soft open subsets of $g_{B}$ is called fuzzy soft interior of $g_{B}$, denoted by,

$F s \operatorname{int}\left(g_{B}\right)=\bigcup\left\{h_{D}, h_{D}\right.$ is fuzzy soft open set and $\left.h_{D} \subseteq g_{B}\right\}$

Definition $2.4^{[7]}$ :A Fuzzy soft set $f_{A}$ in Fuzzy soft topological space $(X, \tau, E)$, is called Fuzzy soft semi-open if $f_{A} \leq F_{S c l} F_{S i n t}\left(f_{A}\right)$, Fuzzy soft semi-closed if $F \operatorname{sint} F \operatorname{Fcl}\left(f_{A}\right) \leq f_{A}$

Definition $2.4^{[12]}$ : A Fuzzy soft set $f_{A}$ in Fuzzy soft topological space $(X, \tau, E)$, is called Fuzzy soft pre-open if $f_{A} \leq F \operatorname{sint} F s c l\left(f_{A}\right)$, Fuzzy soft pre-closed if 
$\operatorname{FsclFsint}\left(f_{A}\right) \leq f_{A}$

Definition $2.4^{[12]}$ : A Fuzzy soft set $f_{A}$ in Fuzzy soft topological space $(X, \tau, E)$, is called Fuzzy soft regular -open if $f_{A}=F \operatorname{sint} F s c l\left(f_{A}\right)$, Fuzzy soft regular-closed if, $F \operatorname{scl} F \operatorname{sint}\left(f_{A}\right)=f_{A}$

Definition $2.5^{[1]}$ : A fuzzy soft set $f_{A}$ in a fuzzy soft topological space $(X, \tau, E)$ is called

--Fuzzy soft b-open set iff $f_{A} \leq\left(\left(F_{S}\right.\right.$ int $\left.\left.F s c l\left(f_{A}\right)\right) \vee\left(F_{S c l} F_{S} \operatorname{int}\left(f_{A}\right)\right)\right)$

--Fuzzy soft b-closed set

iff $f_{A} \geq\left(\left(F_{S}\right.\right.$ int $\left.F S c l\left(f_{A}\right)\right) \vee\left(F_{S C l} F_{S}\right.$ int $\left.\left.\left(f_{A}\right)\right)\right)$

Definition $2.6^{[1]}$ : Let $f_{A}$ be a fuzzy soft set in a fuzzy soft topological space $(X, \tau, E)$ then

-Fuzzy soft b-closure of $f_{A}\left(\mathrm{fsb}-\mathrm{cl}\left(f_{A}\right)\right)$ is defined as ,

fsbcl $\left(f_{A}\right)=\bigcap\left\{g_{B}: g_{B}\right.$ is a fsb-closed set of $\left.X \& g_{B} \geq f_{A}\right\}$

-Fuzzy soft b-interior of $f_{A}\left(\right.$ fsb-int $\left.\left(f_{A}\right)\right)$ is defined as ,

$f s b \operatorname{int}\left(f_{A}\right)=\bigcup\left\{h_{c}: h_{c}\right.$ is a fsb-open set of $\left.X \& h_{C} \leq f_{A}\right\}$

\section{FUZZY SOFT B-OPEN SETS}

Definition 3.1: A Fuzzy soft set $f_{A}$ in Fuzzy soft topological space $(X, \tau, E)$, is called Fuzzy soft semi pre-open if $f_{A} \leq F_{S c l}$ Fsint Fscl $\left(f_{A}\right)$, Fuzzy soft semi preclosed if $F \sin t F s c l F \operatorname{sint}\left(f_{A}\right) \leq f_{A}$

Theorem 3.1: In FSTS X, every Fuzzy soft pre-open set is Fuzzy soft b-open set.

Proof: Let $f_{A}$ be Fuzzy soft pre-open set in X.

$\Rightarrow f_{A} \leq$ Fsint Fscl $\left(f_{A}\right)$

$\Rightarrow f_{A} \leq F \operatorname{sint} F \operatorname{Fcl}\left(f_{A}\right) \vee F \operatorname{Fsl} F \operatorname{sint}\left(f_{A}\right)$

$\Rightarrow f_{A}$ is fuzzy soft b-open set in $\mathrm{X}$.

But converse is not true as seen from the below example.

Let $\tau=\left\{\tilde{0}, \tilde{1},\left(F_{1}, E\right),\left(F_{2}, E\right)\right\}$

$$
\left(F_{1}, E\right)=\left\{\left\{\frac{1}{5}, \frac{1}{4}\right\},\left\{0, \frac{1}{3}\right\}\right\}, \quad\left(F_{2}, E\right)=\left\{\left\{\frac{1}{3}, \frac{1}{2}\right\},\left\{\frac{1}{4}, \frac{1}{2}\right\}\right\}
$$


Let $f_{A}=\left\{\left\{\frac{1}{5}, \frac{1}{3}\right\},\left\{\frac{3}{4}, \frac{1}{3}\right\}\right\}$

$f_{A}$ is fuzzy soft b-open set but not fuzzy soft pre-open as $f_{A} \geq F \sin t \operatorname{Fscl}\left(f_{A}\right)$.

\section{Remarks:}

(i) If $f_{A}$ is Fuzzy soft subset of FSTS $(X, \tau, E)$, then

$\operatorname{Fsbcl}\left(f_{A}\right)$ is the smallest fuzzy soft b-closed set containing $f_{A}$,

$\Rightarrow \operatorname{Fsbcl}\left(f_{A}\right)=f_{A} \vee\left[F \sin t F \operatorname{scl}\left(f_{A}\right) \wedge F \operatorname{scl} F \sin t\left(f_{A}\right)\right.$

(ii) $F s b \operatorname{int}\left(f_{A}\right)$ is the largest fuzzy soft b-closed set contained in $f_{A}$,

$\Rightarrow F \operatorname{sint}\left(f_{A}\right)=f_{A} \wedge\left[F \sin t F \operatorname{scl}\left(f_{A}\right) \vee F S c l F \sin t\left(f_{A}\right)\right]$

Theorem 3.2 : In FSTS X, every Fuzzy soft b-open set is Fuzzy soft semi pre-open.

Proof: Let $f_{A}$ be Fuzzy soft b-open set in X.

$\Rightarrow f_{A} \leq\left(\left(F_{S}\right.\right.$ int $\left.\left.F_{S c l}\left(f_{A}\right)\right) \vee\left(F_{S c l} F_{S} \operatorname{int}\left(f_{A}\right)\right)\right)$

Since $\operatorname{Fscl}\left(f_{A}\right)$ is the smallest fuzzy soft closed set containing $f_{A}, f_{A} \leq \operatorname{Fscl}\left(f_{A}\right)$

$f_{A} \leq\left(\left(F_{S}\right.\right.$ int $\left.\operatorname{Fscl}\left(f_{A}\right)\right) \vee\left(F s c l\right.$ Fs int $\left.\left.F s c l\left(f_{A}\right)\right)\right)$

$\Rightarrow f_{A} \leq\left(F s c l F \operatorname{int} F \operatorname{scl}\left(f_{A}\right)\right)$

$\Rightarrow f_{A}$ is Fuzzy soft semi-pre open set in X.

But converse is not true as seen from the below example.

Let $\tau=\left\{\tilde{0}, \tilde{1},\left(F_{1}, E\right),\left(F_{2}, E\right)\right\}$

$$
\left(F_{1}, E\right)=\left\{\left\{\frac{1}{2}, \frac{1}{3}\right\},\left\{\frac{1}{3}, \frac{1}{5}\right\}\right\} \quad\left(F_{2}, E\right)=\left\{\left\{\frac{1}{2}, \frac{2}{3}\right\},\left\{\frac{1}{3}, \frac{1}{4}\right\}\right\} \quad \text { Let }
$$

$k=\left\{\left\{\frac{1}{3}, \frac{1}{5}\right\},\left\{\frac{1}{3}, \frac{1}{4}\right\}\right\}$

$k$ is fuzzy soft semi pre-open but not fuzzy soft b-open set.

Theorem 3.3 : (i) In a FSTS, a fuzzy soft set $f_{A}$ is fuzzy soft b-closed iff $f_{A}=\operatorname{Fsbcl}\left(f_{A}\right)$

(ii) In a FSTS, a fuzzy soft set $f_{A}$ is fuzzy soft b-open iff $f_{A}=F \operatorname{sinint}\left(f_{A}\right)$

Proof: (i) Let $f_{A}=\operatorname{Fs} b c l\left(f_{A}\right)$

$\Rightarrow f_{A}=\wedge\left\{g_{B} ; g_{B}\right.$ is Fs $b-$ closed set and $\left.f_{A} \leq g_{B}\right\}$ 
$\Rightarrow f_{A} \in \wedge\left\{g_{B} ; g_{B}\right.$ is Fsb-closed set and $\left.f_{A} \leq g_{B}\right\}$

$\Rightarrow f_{A}$ is Fsb-closed set.

Conversely, let $f_{A}$ be fuzzy soft b-closed set.

$\Rightarrow f_{A} \in \wedge\left\{g_{B} ; g_{B}\right.$ is Fsb-closed set $\}$ and $f_{A} \leq f_{A}$

$\Rightarrow f_{A}$ is the smallest closed set containing $f_{A}$.

$\Rightarrow f_{A}=\wedge\left\{g_{B} ; g_{B}\right.$ is Fs $b-$ closed set and $\left.f_{A} \leq g_{B}\right\}$

Thus $f_{A}=\operatorname{Fsbcl}\left(f_{A}\right)$

(ii) Let $f_{A}=F s b \operatorname{int}\left(f_{A}\right)$

Then $f_{A}$ is fuzzy soft b-open set.

If $f_{A}$ is fuzzy soft b-open set, then $f_{A} \in\left\{g_{B} ; g_{B}\right.$ is Fsb-open set $\}$ and $f_{A} \leq f_{A}$

$f_{A} \in\left\{g_{B} ; g_{B}\right.$ is Fsb-open and $\left.g_{B} \leq f_{A}\right\}$

$\Rightarrow f_{A}=F s b \operatorname{int}\left(f_{A}\right)$

Theorem3.4: In a FSTS,

(i) $\operatorname{Fsbcl}(A \vee B) \geq F \operatorname{sbcl}(A) \vee F \operatorname{sbcl}(B)$

(ii) $\operatorname{Fsbcl}(A \wedge B) \geq F \operatorname{sbcl}(A) \wedge F \operatorname{sbcl}(B)$

(iii) $F s b \operatorname{int}(A \vee B) \geq F s b \operatorname{int}(A) \vee F_{S} b \operatorname{int}(B)$

(iv) $F s b \operatorname{int}(A \wedge B) \leq F s b \operatorname{int}(A) \wedge F s b \operatorname{int}(B)$

Proof: Let A and B be any two fuzzy soft sets in FSTS $(X, \tau, E)$

(i) $A \leq A \vee B \Rightarrow F \operatorname{sicl}(A) \leq F \operatorname{sbcl}(A \vee B)$

$B \leq A \vee B \quad \Rightarrow \quad F \operatorname{sbcl}(B) \leq F \operatorname{sbcl}(A \vee B)$

$\operatorname{Fs} b c l(A) \vee F_{S} b c l(B) \leq F \operatorname{Fscl}(A \vee B)$

(ii) $A \wedge B \leq A$ and $A \wedge B \leq B \Rightarrow \operatorname{Fsbcl}(A \wedge B) \leq F \operatorname{sbcl}(A) \wedge F \operatorname{sicl}(B)$

(iii) Let A and B be any two fuzzy soft sets in FSTS $(X, \tau, E)$

$A \leq A \vee B \Rightarrow F s b \operatorname{int}(A) \leq F s b \operatorname{int}(A \vee B)$

$B \leq A \vee B \Rightarrow F s b \operatorname{int}(B) \leq F \operatorname{sint}(A \vee B)$

$F s b \operatorname{int}(A) \vee F s b \operatorname{int}(B) \leq F s b \operatorname{int}(A \vee B)$

(iv) $A \wedge B \leq A$ and $A \wedge B \leq B \Rightarrow F_{S} b \operatorname{int}(A \wedge B) \leq F_{S} b \operatorname{int}(A) \wedge F_{S} b \operatorname{int}(B)$ 
Theorem 3.5: Let $f_{A}$ be a fuzzy soft b-open set in $X$,

(i) If $f_{A}$ is fuzzy soft regularly closed set then $A$ is fuzzy soft pre-open set.

(ii) If $f_{A}$ is fuzzy soft regularly open set then $A$ is fuzzy soft semi-open set.

Proof: Let $f_{A}$ be fuzzy soft b-open set in X.

$f_{A} \leq\left(\left(F_{S}\right.\right.$ int $\left.F s c l\left(f_{A}\right)\right) \vee\left(F s c l F s\right.$ int $\left.\left.F s c l\left(f_{A}\right)\right)\right)$

(i) If $f_{A}$ is fuzzy soft regularly closed set then $f_{A}=F s c l F \sin t\left(f_{A}\right)$

$\Rightarrow f_{A} \leq\left(\left(F S\right.\right.$ int $\left.\left.\left.\operatorname{Fscl}\left(f_{A}\right)\right) \vee\left(f_{A}\right)\right)\right)$

$\Rightarrow f_{A} \leq\left(F S\right.$ int $\left.\operatorname{Fscl}\left(f_{A}\right)\right)$

$\Rightarrow f_{A}$ is fuzzy soft pre-open set.

(ii) If $f_{A}$ is fuzzy soft regularly open set then $f_{A}=F \sin t F \operatorname{sicl}\left(f_{A}\right)$

$\Rightarrow f_{A} \leq$ FsclFs int $\left.\left(f_{A}\right)\right) \vee\left(f_{A}\right)$

$\Rightarrow f_{A} \leq F_{S C l F S}$ int $\left(f_{A}\right)$

$\Rightarrow f_{A}$ is fuzzy soft semi-open set.

Theorem 3.6: Let $f_{A}$ be a fuzzy soft b-open set in $X$,

(i) If $f_{A}$ is fuzzy soft regularly closed set then $A$ is fuzzy soft semi-closed set.

(ii) If $f_{A}$ is fuzzy soft regularly open set then $A$ is fuzzy soft pre-closed set.

Proof: Let $f_{A}$ be fuzzy soft b-open set in X.

$f_{A} \leq\left(\left(F_{S}\right.\right.$ int $\left.F s c l\left(f_{A}\right)\right) \vee\left(F s c l F s\right.$ int $\left.\left.F s c l\left(f_{A}\right)\right)\right)$

(i) If $f_{A}$ is fuzzy soft regularly closed set then $f_{A}=F s c l F \sin t\left(f_{A}\right)$

$\Rightarrow f_{A}=\left(\left(F S\right.\right.$ int $\left.\left.\left.\operatorname{Fscl}\left(f_{A}\right)\right) \vee\left(f_{A}\right)\right)\right)$

$\Rightarrow f_{A}=\left(F S\right.$ int $\left.F S C l\left(f_{A}\right)\right)$

$\Rightarrow\left(F_{S}\right.$ int $\left.F s c l\left(f_{A}\right)\right) \leq f_{A}$

$\Rightarrow f_{A}$ is fuzzy soft semi-closed set.

(ii) If $f_{A}$ is fuzzy soft regularly open set then $f_{A}=F \sin t F \operatorname{sicl}\left(f_{A}\right)$

$\Rightarrow f_{A}=F S c l F S$ int $\left.\left(f_{A}\right)\right) \vee\left(f_{A}\right)$

$\Rightarrow$ FsclFs int $\left(f_{A}\right) \leq f_{A}$ and $f_{A} \leq f_{A}$

$\Rightarrow f_{A}$ is fuzzy soft pre-closed set. 
Theorem 3.7: (i) For any fuzzy soft b-closed set $f_{A}$ in FSTS $X$, Fs int $\left(f_{A}\right)$ is fuzzy soft regular open set.

(ii) For any fuzzy soft b-open set $f_{A}$ in FSTS $X$, Fscl $\left(f_{A}\right)$ is fuzzy soft regular closed set.

Proof: (i) Let $f_{A}$ be fuzzy soft b-closed set in

$\mathrm{X} . \Rightarrow\left(F_{s}\right.$ int $F s c l\left(f_{A}\right) \wedge\left(F s c l F s\right.$ int $\left.\left(f_{A}\right)\right) \leq f_{A}$

$\Rightarrow F_{S}$ int $\left(F_{S}\right.$ int $\left.F_{S c l}\left(f_{A}\right)\right) \wedge F_{S} \operatorname{int}\left(F_{S c l} F_{S} \operatorname{int}\left(f_{A}\right)\right) \leq F_{S} \operatorname{int}\left(f_{A}\right)$

$\Rightarrow F_{S} \operatorname{int}\left(F_{S C l} F_{S} \operatorname{int}\left(f_{A}\right)\right) \leq F_{S} \operatorname{int}\left(f_{A}\right)$ Also

$F_{S} \operatorname{int}\left(f_{A}\right) \leq F_{S} \operatorname{int}\left(F_{S C l} F_{S} \operatorname{int}\left(f_{A}\right)\right) \therefore F_{S} \operatorname{int}\left(f_{A}\right)=F_{S} \operatorname{int}\left(F_{S C l} F_{S} \operatorname{int}\left(f_{A}\right)\right)$,

Fs int $\left(f_{A}\right)$ is fuzzy soft regular open set.

(ii) Let $f_{A}$ be fuzzy soft b-open set in $\mathrm{X}$.

$\Rightarrow\left(F_{S}\right.$ int $F s c l\left(f_{A}\right) \vee\left(F S c l\right.$ Fs int $\left.\left(f_{A}\right)\right) \geq f_{A}$

$\Rightarrow \operatorname{Fscl}\left(F_{S}\right.$ int $\left.F s c l\left(f_{A}\right)\right) \vee \operatorname{Fscl}\left(F s c l F_{S} \operatorname{int}\left(f_{A}\right)\right) \geq \operatorname{Fscl}\left(f_{A}\right)$

$\Rightarrow \operatorname{Fscl}\left(F \operatorname{int} F s c l\left(f_{A}\right)\right) \geq \operatorname{Fscl}\left(f_{A}\right)$ Also

$\operatorname{Fscl}\left(f_{A}\right) \geq \operatorname{Fscl}\left(F_{S}\right.$ int $\left.F_{S c l}\left(f_{A}\right)\right) \therefore \operatorname{Fscl}\left(f_{A}\right)=\operatorname{Fscl}\left(F_{S}\right.$ int $\left.F_{S c l}\left(f_{A}\right)\right), \operatorname{Fscl}\left(f_{A}\right)$ is fuzzy soft regular closed set.

Theorem 3.8: (i) Let $f_{A}$ be fuzzy soft b-open set in FSTS X, such that $F \sin t\left(f_{A}\right)=0$ then $f_{A}$ is fuzzy soft pre-open set.

(ii) Let $f_{A}$ be fuzzy soft b-open set in FSTS X, such that Fscl $\left(f_{A}\right)=0$ then $f_{A}$ is fuzzy soft semi-open set.

Proof: (i) Let $f_{A}$ be fuzzy soft b-open set in X.

$\Rightarrow\left(F s\right.$ int $F s c l\left(f_{A}\right) \vee\left(F S c l F s\right.$ int $\left.\left(f_{A}\right)\right) \geq f_{A}$ and $F \sin t\left(f_{A}\right)=0$, therefore Fscl F $\sin t\left(f_{A}\right)=0$

$\Rightarrow F_{S}$ int $\operatorname{Fscl}\left(f_{A}\right) \geq f_{A}$. Thus $f_{A}$ is fuzzy soft pre-open set.

(ii) Let $f_{A}$ be fuzzy soft b-open set in $\mathrm{X}$.

$\Rightarrow\left(F_{S}\right.$ int $F_{S C l}\left(f_{A}\right) \vee\left(F_{S c l} F_{S} \operatorname{int}\left(f_{A}\right)\right) \geq f_{A}$ and $\operatorname{Fscl}\left(f_{A}\right)=0$, therefore $F \sin t$ Fscl $\left(f_{A}\right)=0$

$\Rightarrow$ Fscl Fs int $\left(f_{A}\right) \geq f_{A}$, Thus $f_{A}$ is fuzzy soft semi-open set.

Theorem 3.9: If $f_{A}$ is fuzzy soft open set and $g_{B}$ is fuzzy soft b-open set in FSTS X, 
then $f_{A} \wedge g_{B}$ is fuzzy soft b-open set.

Proof: Consider $f_{A} \wedge g_{B} \leq f_{A} \wedge F \operatorname{sclF} \sin t\left(g_{B}\right) \vee F \sin t F s c l\left(g_{B}\right)$

$f_{A} \wedge g_{B}=f_{A} \wedge F s c l F \sin t\left(g_{B}\right) \vee f_{A} \wedge F \sin t F s c l\left(g_{B}\right)$

$f_{A} \wedge g_{B}=\left[F s c l F \sin t\left(f_{A}\right) \wedge F s c l F \sin t\left(g_{B}\right)\right] \vee\left[F \sin t F s c l\left(f_{A}\right) \wedge F s c l F \sin t\left(g_{B}\right)\right]$

$f_{A} \wedge g_{B}=F \operatorname{sclF} \sin t\left(f_{A} \wedge g_{B}\right) \vee F \sin t F s c l\left(f_{A} \wedge g_{B}\right)$

$f_{A} \wedge g_{B}$ is fuzzy soft b-open set in X.

\section{FUZZY SOFT B-CONTINUOUS FUNCTIONS}

Definition 4.1: A function $f:(X, \tau) \rightarrow\left(Y, \tau^{\prime}\right)$ is said to be fuzzy soft $\mathrm{b}$ - continuous (FSb- continuous) if for every fuzzy soft open set $(\mathrm{G}, \mathrm{E}) \in \tau^{\prime}, f^{-1}(G, E)$ is fuzzy soft b-open set in $\tau$.

Theorem 4.1: Every fuzzy soft continuous function is Fuzzy soft b-continuous function.

Proof: Let $f: X \rightarrow Y$ be Fuzzy soft continuous function. i.e Inverse of every fuzzy soft open set is open in $X$. Since every fuzzy soft open set of $X$ is fuzzy soft b-open, $f$ is fuzzy soft b-continuous. But converse need not be true. Consider the following example.

Let $f:(X, \tau) \rightarrow\left(Y, \tau^{\prime}\right)$ defined by $f\left(x_{1}\right)=y_{2}$ and $f\left(x_{2}\right)=y_{1}$

$\tau=\left\{\tilde{0}, \tilde{1},\left(F_{1}, E\right),\left(F_{2}, E\right),\left(F_{3}, E\right),\left(F_{4}, E\right),\left(F_{5}, E\right),\left(F_{6}, E\right),\left(F_{7}, E\right)\right\}$

$\tau^{\prime}=\left\{\tilde{0}, \tilde{1},\left(G_{1}, E\right),\left(G_{2}, E\right)\right\}$ be fuzzy soft topological spaces.

$$
\begin{aligned}
& \text { Let } X=\left\{x_{1}, x_{2}\right\} Y=\left\{y_{1}, y_{2}\right\} E=\left\{e_{1}, e_{2}\right\}\left(F_{1}, E\right)=\left\{\left\{\frac{1}{2}, \frac{1}{3}\right\},\left\{\frac{1}{4}, \frac{2}{3}\right\}\right\} \\
& \left(F_{2}, E\right)=\left\{\left\{\frac{1}{3}, \frac{1}{4}\right\},\left\{0, \frac{1}{6}\right\}\right\}\left(F_{3}, E\right)=\left\{\left\{\frac{1}{2}, 1\right\},\left\{\frac{2}{3}, \frac{1}{6}\right\}\right\}\left(F_{4}, E\right)=\left\{\left\{\frac{1}{5}, \frac{1}{3}\right\},\left\{\frac{1}{4}, \frac{1}{6}\right\}\right\} \\
& \left(F_{5}, E\right)=\left\{\left\{\frac{1}{5}, \frac{1}{4}\right\},\left\{0, \frac{1}{6}\right\}\right\}\left(F_{6}, E\right)=\left\{\left\{\frac{1}{2}, 1\right\},\left\{\frac{2}{3}, \frac{2}{3}\right\}\right\}\left(F_{7}, E\right)=\left\{\left\{\frac{1}{3}, \frac{1}{3}\right\},\left\{\frac{1}{4}, \frac{1}{6}\right\}\right\} \\
& \left(G_{1}, E\right)=\left\{\left\{\frac{1}{2}, \frac{1}{4}\right\},\left\{\frac{1}{5}, 0\right\}\right\} \quad\left(G_{2}, E\right)=\left\{\left\{\frac{1}{4}, \frac{1}{5}\right\},\left\{\frac{1}{6}, 0\right\}\right\}
\end{aligned}
$$


$f^{-1}\left(G_{1}, E\right)=\left\{\left\{\frac{1}{4}, \frac{1}{2}\right\}\left\{0, \frac{1}{5}\right\}\right\} \quad f^{-1}\left(G_{2}, E\right)=\left\{\left\{\frac{1}{5}, \frac{1}{4}\right\}\left\{0, \frac{1}{6}\right\}\right\}$

$f^{-1}\left(G_{1}, E\right)$ and $f^{-1}\left(G_{2}, E\right)$ are Fuzzy soft b-open sets in X. But $f^{-1}\left(G_{1}, E\right)$ is not fuzzy soft open set in $X$.

Therefore $\mathrm{f}$ is fuzzy soft b-continuous function but not fuzzy soft continuous.

Definition 4.2: A fuzzy soft set A of a FSTS $(X, \tau)$ is called Fuzzy soft generalized $\mathrm{b}$-closed set if $\operatorname{Fsbcl}(A) \leq B$ whenever $A \leq B$ and $\mathrm{B}$ is fuzzy soft open set in $(X, \tau)$.

Theorem 4.2: Every fuzzy soft b-open (b-closed) set is fuzzy soft generalized b-open (generalized b-closed) .

Proof: By definition every fuzzy soft b-open set is generalized b-open.

But converse is not true.

Example: $\tau=\{\tilde{0}, \tilde{1}, A=\{1,0.6\}\}$

$f_{s b 0}(X)=\{\tilde{0}, \tilde{1}, A=\{1,0.6\},\{\alpha, \beta)\}$ where $\alpha \succ 0$ or $\beta \succ 0.4$

$f_{s} b c(X)=\{\tilde{0}, \tilde{1}, B=\{0,0.4\},\{\alpha, \beta)\}$ where $\alpha=0$ and $\beta \prec 0.4$

Consider $H=\{0,0.3\}$ which is fuzzy soft generalized b-open set but not fuzzy soft bopen.

Theorem 4.3: If $f_{A}$ is Fsg- $b$ closed and fuzzy soft b-open set then it is fuzzy soft $b$ closed.

Proof: Let $f_{A}$ is Fsg- b closed and fuzzy soft b-open set. Then $f_{A} \leq f_{A}$ and $\operatorname{Fsbcl}\left(f_{A}\right) \leq f_{A}$

But $f_{A} \leq \operatorname{Fsbcl}\left(f_{A}\right)$, therefore $f_{A}=\operatorname{Fsbcl}\left(f_{A}\right), f_{A}$ is fuzzy soft b-closed.

Definition 4.3: A function $f:(X, \tau) \rightarrow\left(Y, \tau^{\prime}\right)$ is said to be fuzzy soft generalized-b continuous if inverse image of every fuzzy soft closed set in $Y$ is fuzzy soft generalized b-closed set in X.

Theorem 4.4: A function $f:(X, \tau) \rightarrow\left(Y, \tau^{\prime}\right)$ is Fuzzy soft generalized $b$-continuous iff the inverse image of each fuzzy soft open set of $Y$ is fuzzy soft generalized $b$ - open set of $X$.

Proof: Let B be fuzzy soft open set in Y. Then 1 - B is fuzzy sof closed in Y.

Since $\mathrm{f}$ is fuzzy soft $\mathrm{gb}$ - continuous, $f^{-1}(1-B)=1-f^{-1}(B)$ is fuzzy soft $\mathrm{g}$-b closed 
in $X$.

$\Rightarrow f^{-1}(B)$ is fuzzy soft gb- open set of $\mathrm{X}$. Hence the theorem and converse is obvious.

\section{Remarks}

(i) Every fuzzy soft open (closed) set is fuzzy soft b - open (closed) set.

(ii) Every fuzzy soft b - closed (open ) set is Fuzzy soft generalized b - closed (open ) set.

(iii) Every fuzzy soft open (closed) set is fuzzy soft gb-open (closed) set.

Theorem 4.5: Every fuzzy soft continuous function is Fuzzy soft generalized b continuous (FSgb - continuous) function.

Proof: Let $f:(X, \tau) \rightarrow\left(Y, \tau^{\prime}\right)$ be fuzzy soft continuous function. Then inverse of fuzzy soft open set is fuzzy soft open in X. Since every fuzzy soft open set is fuzzy soft gb-open, the function $\mathrm{f}$ is FSgb-continuous. But converse need not be true as seen from the following example.

Let $f:(X, \tau) \rightarrow\left(Y, \tau^{\prime}\right)$ defined by $f\left(x_{1}\right)=y_{1}$ and $f\left(x_{2}\right)=y_{2}$

$\tau=\{\tilde{0}, \tilde{1}, A=\{1,0.9\}\} \quad \tau^{\prime}=\{\tilde{0}, \tilde{1}, B=\{0,0.1\}\}$

$f_{s} b o(X)=\{\tilde{0}, \tilde{1},\{1,0.9\},\{\alpha, \beta\}\} \quad$ where $\alpha \succ 0$ or $\beta \succ 0.1$

$f_{s b c}(X)=\{\tilde{0}, \tilde{1},\{0,0.1\},\{\alpha, \beta\}\} \quad$ where $\alpha=0$ or $\beta \prec 0.1$

$f^{-1}(B)=\{0,0.05\}$ is not fuzzy soft open set in X.

Thus $\mathrm{f}$ is not fuzzy soft continuous, But $\{0,0.05\}$ is FSgb-open set in $\mathrm{X}$. Thus $\mathrm{f}$ is FSgb-continuous function.

Theorem 4.6: Every fuzzy soft b-continuous function is fuzzy soft generalized b continuous function.

Proof: Let $f:(X, \tau) \rightarrow\left(Y, \tau^{\prime}\right)$ be fuzzy soft b-continuous function. Let A be fuzzy soft open set in $\mathrm{Y}$. $f^{-1}(A)$ is fuzzy soft b-open in $\mathrm{X}$, since $\mathrm{f}$ is FS b - continuous.

$1-f^{-1}(A)=f^{-1}(1-A)$ is FS b-closed set in $\mathrm{X}$ for every 1 -A closed set in Y. Hence $f^{-1}(1-A)$ is fsgb - closed in $\mathrm{X}$ and $\mathrm{f}$ is FS gb-continuous. Converse need not be true.

consider the following example:-

Let $f:(X, \tau) \rightarrow\left(Y, \tau^{\prime}\right)$ be an identity mapping.

$\tau=\{\tilde{0}, \tilde{1}, A=\{1,0.6\}\}$

$\tau^{\prime}=\{\tilde{0}, \tilde{1}, B=\{0,0.3\}\}$ 
$f_{s} b 0(X)=\{\tilde{0}, \tilde{1}, A=\{1,0.6\},\{\alpha, \beta)\}$ where $\alpha \succ 0$ or $\beta \succ 0.4$

$f_{s} b c(X)=\{\tilde{0}, \tilde{1}, B=\{0,0.3\},\{\alpha, \beta)\}$ where $\alpha=0$ and $\beta \prec 0.4$

$f^{-1}(B)=\{0,0.3\}$ is not FS b-open set in X. But it is FS gb-open in X.

Definition 4.4: A function $f:(X, \tau) \rightarrow\left(Y, \tau^{\prime}\right)$ is said to be FS generalised $b$ Irresolute if $f^{-1}(A)$ is FS gb-closed set in Xfor every FS gb-closed set $A$ in $Y$.

Theorem 4.7: A mapping $f:(X, \tau) \rightarrow\left(Y, \tau^{\prime}\right)$ is FSgb-irresolute if and only if inverse image of every gb-open FS set in $Y$ is gb-open FS set in $X$.

proof is obvious.

Theorem 4.8: Every fuzzy soft gb-irresolute mapping is Fuzzy soft gb-continuous mapping.

Proof: Let $f: X \rightarrow Y$ is fsgb-irresolute. Let $\mathrm{V}$ be a closed fuzzy soft set in $\mathrm{Y}$, Then $\mathrm{V}$ is

FSgb-closed set in Y. Since $\mathrm{f}$ is fsgb-irresolute, $f^{-1}(V)$ is gb-closed fuzzy soft set in $\mathrm{X}$.

Hence $\mathrm{f}$ is fsgb-continuous mapping. Converse need not be true as seen from the following example.

Let $f:(X, \tau) \rightarrow\left(Y, \tau^{\prime}\right)$ be an identity mapping.

Where $\tau=\{\tilde{0}, \tilde{1},\{0.6,0.7\},\{0.6,0.2\}\}, \tau^{\prime}=\{\tilde{0}, \tilde{1},\{0.7,0.7\}\}$

Consider a fsgb-closed set $A=\{0.1,0.1\}$ in Y.

$\operatorname{FSbo}(X)=\{\tilde{0}, \tilde{1},\{0.6,0.7\},\{0.6,0.2\},\{\alpha, \beta\}\}$ where $\alpha \succ 0$ or $\beta \succ 0.3$

$\operatorname{FSbc}(X)=\{\tilde{0}, \tilde{1},\{0.4,0.3\},\{\alpha, \beta\}\}$ where $\alpha=0$ and $\beta \prec 0.3$

$f^{-1}(A)=\{0.1,0.1\}$ is not fsgb-closed set in $\mathrm{X}$, hence $\mathrm{f}$ is not FSgb-irresolute mapping.

Since inverse image of every FS open set in $\mathrm{Y}$ is FS gb-open set in $\mathrm{X}, t$ is fsgbcontinuous mapping.

Theorem 4.9: Let $f:(X, \tau) \rightarrow\left(Y, \tau^{\prime}\right)$ and $g:\left(Y, \tau^{\prime}\right) \rightarrow(Z, \sigma)$ be two functions. Then

(i) $g \bullet f: X \rightarrow Z$ is $f$ sgb-continuous, if $f$ is $f s g b$ - continuous and $g$ is fuzzy soft continuous. 
(ii) $g \bullet f: X \rightarrow Z$ is fsgb-irresolute, iff and $g$ are $f s g b$-irresolute functions.

(iii) $g \bullet f: X \rightarrow Z$ is $f$ sgb-continuous iff is $f$ sgb-irresolute and $g$ is $f$ sgb-continuous.

Proof: (i) Let A be fuzzy soft closed subset of Z. Then $g^{-1}(A)$ is fuzzy soft closed set of $\mathrm{Y}$, since $g:\left(Y, \tau^{\prime}\right) \rightarrow(Z, \sigma)$ is fuzzy soft continuous and also $f^{-1}\left(g^{-1}(A)\right)=(g \bullet f)^{-1}(A)$ is fsgb-closed in $X$, Since $f:(X, \tau) \rightarrow\left(Y, \tau^{\prime}\right)$ is fsgbcontinuous. Hence $g \bullet f: X \rightarrow Z$ is fsgb-continuous.

(ii) Let $g:\left(Y, \tau^{\prime}\right) \rightarrow(Z, \sigma)$ be fsgb-irresolute and let A be fsgb-closed subset of $\mathrm{Z}$.

$g^{-1}(A)$ is fsgb-closed set of $\mathrm{Y}$, Since $\mathrm{g}$ is fsgb-irresolute function. Also $f:(X, \tau) \rightarrow\left(Y, \tau^{\prime}\right)$ is fsgb-irresolute, $f^{-1}\left(g^{-1}(A)\right)=(g \bullet f)^{-1}(A)$ is fsgb-closed set. Thus $g \bullet f: X \rightarrow Z$ is fsgb-irresolute function.

(iii) Let $\mathrm{A}$ be fsb-closed subset of, $g^{-1}(A)$ is fuzzy soft gb-closed subset of Y. Since $g:\left(Y, \tau^{\prime}\right) \rightarrow(Z, \sigma)$ is fsgb-continuous. Also, $\mathrm{f}$ is fsgb-irresolute, so every fsgb-closed set of $\mathrm{Y}$ is fsgb-closed in X. Thus $f^{-1}\left(g^{-1}(A)\right)=(g \bullet f)^{-1}(A)$ is fsgb-closed set of $\mathrm{X}$. Hence $g \bullet f: X \rightarrow Z$ is fsgb-continuous.

Definition 4.5: A function $f:(X, \tau) \rightarrow\left(Y, \tau^{*}\right)$ is Fs $b^{*}$ - continuous if inverse of fuzzy soft b-open set is Fsb-open in X.

Theorem 5: Every Fs $b^{*}$-continuous function is b-continuous, but converse is not true.

Proof: Since every Fuzzy soft open set is Fuzzy soft b-open set theorem is obvious but converse is not true .

Consider a function $f: X \rightarrow Y$ defined by $f\left(x_{1}\right)=y_{2} \quad \& \quad f\left(x_{2}\right)=y_{2}$

$$
\begin{aligned}
\tau & =\left\{\tilde{0}, \tilde{1},\left(F_{1}, E\right),\left(F_{2}, E\right)\right\} \\
\tau^{*} & =\left\{\tilde{0}, \tilde{1},\left(G_{1}, E\right),\left(G_{2}, E\right)\right\}
\end{aligned}
$$

Where $\left(F_{1}, E\right)=\left\{\left\{\frac{1}{2}, \frac{1}{3}\right\},\left\{\frac{1}{3}, \frac{1}{5}\right\}\right\} \quad\left(F_{2}, E\right)=\left\{\left\{\frac{1}{2}, \frac{2}{3}\right\},\left\{\frac{1}{3}, \frac{1}{4}\right\}\right\}$

$$
\begin{array}{ll}
\left(G_{1}, E\right)=\left\{\left\{\frac{1}{4}, \frac{1}{3}\right\},\left\{0, \frac{1}{3}\right\}\right\} & \left(G_{2}, E\right)=\left\{\left\{\frac{1}{3}, \frac{1}{2}\right\},\left\{\frac{1}{4}, \frac{1}{2}\right\}\right\} \\
f^{-1}\left(G_{1}\right)=\left\{\left\{\frac{1}{3}, \frac{1}{4}\right\},\left\{\frac{1}{3}, 0\right\}\right\}=A & f^{-1}\left(G_{2}\right)=\left\{\left\{\frac{1}{2}, \frac{1}{3}\right\},\left\{\frac{1}{2}, \frac{1}{4}\right\}\right\}=B
\end{array}
$$

$\mathrm{A}$ and $\mathrm{B}$ are fuzzy soft $\mathrm{b}$-open sets in $\mathrm{X}$. Therefore $\mathrm{f}$ is fuzzy soft $\mathrm{b}$ - continuous. 
Consider a fuzzy soft b-open set $H=\left\{\left\{\frac{1}{5}, \frac{1}{3}\right\},\left\{\frac{1}{4}, \frac{1}{3}\right\}\right\}$ in $\mathrm{Y}$. $f^{-1}(H)=\left\{\left\{\frac{1}{3}, \frac{1}{5}\right\},\left\{\frac{1}{3}, \frac{1}{4}\right\}\right\}=K$

$\mathrm{K}$ is not Fuzzy soft b-open in $\mathrm{X}$. Therefore $\mathrm{f}$ is not Fuzzy soft $b^{*}$ - continuous.

\section{CONCLUSION}

In this paper we have introduced fuzzy soft semi pre-open set and its relationship with fuzzy soft b-open set is studied. And we introduced fuzzy soft b-continuous functions, generalized $b$-continuous functions, generalized $b$-irresolute functions, $b^{*}$ - continuous functions and then studied some properties of these functions.

\section{REFERENCES}

[1] P. N Anil "Fuzzy soft B-open sets in Fuzzy soft topological space", International Journal of science, technology \& Engineering, Vol. 3, Issue 02, Aug 2016.

[2] S.S Benchalli and K.Jenifer."On Fuzzy b-open sets in fuzzy soft topological space", Journal of Computer and Mathematical Science, Vol.1(2), pp.127134, 2010.

[3] S. S.Benchalli and Jenifer J.Karnel “ Fuzzy gb-Continuous Maps in Fuzzy Topological Spaces”, International Journal of Computer Applications (0975 -8887) Vol 19 No.1, April 2011.

[4] C L.Chang, "Fuzzy topological spaces", Journal of Mathematical Analysis and Applications, vol. 24, pp.182190,1968.

[5] P.K Gain, P.Mukherjee, R.P Chakraborty and M.Pal, "On some structural properties of fuzzy soft topological spaces", International Journal of Fuzzy Mathematical Archive. Vol.1, pp. 1 - 15, 2013.

[6] C Gunduz and S Bayramov, "Mathematical Problems in Engineering", Vol.2013, Article ID 835308, 1pages, 2013.

[7] A.Kandil, O.A.E Tantawy, S.A. El-Sheikh and A.M.Abd El-latif. " Fuzzy semi open soft sets related properties in fuzzy soft topological spaces", Journal of mathematics and computer science, 13:94-114, 2014.

[8] A. Kharal and B. Ahmad, "Mappings on fuzzy soft classes", Advances in Fuzzy Systems, vol. 2009, Article ID 407890, 6 pages, 2009.

[9] P. K. Maji, R. Biswas, and A. R. Roy, "Fuzzy soft sets," Journal of Fuzzy Mathematics, vol.9 no. 3, pp. 589-602, 2001. 
[10] D. Molodtsov, "Soft set theory-first results", Computers \& Mathematics with applications, vol. 37, no. 4-5, pp. 19-31, 1999.

[11] S. Roy and T. K. Samanta, "A note on fuzzy soft topological spaces", Annals of fuzzy mathematics and informatics, vol. 3, no. 2, pp. 305-311, 2012

[12] Sabir hussain "On weak and strong forms of fuzzy soft open sets", Fuzzy Information and Engineering, vol 8, issue 4, ,pp 451-463, Dec 2016.

[13] B. Tanay and M. B. Kandemir, "Topological structure of fuzzy soft sets", Computers \& Mathematics with Applications, vol. 61, no. 10, pp. 29522957, 2011.

[14] B. P.Varol and H. Aygün, "Fuzzy soft topology", Hacettepe Journal of Mathematics and Statistics, vol. 41, no. 3, pp. 407-419, 2012.

[15] L. A. Zadeh, "Fuzzy sets", Information and Control, vol. 8, no. 3, pp. 338$353,1965$. 